\title{
The short-time behavior of kinetic spherical model with long-ranged interactions *
}

\author{
Yuan Chen ${ }^{a}$,Shuohong Guo ${ }^{a}$, Zhibing $\mathrm{Li}^{a, b}$ and Aijun Ye ${ }^{a}$ \\ ${ }^{a}$ Department of Physics, Zhongshan University, Guangzhou 510275, China \\ ${ }^{b}$ Associate Member of the ICTP, Italy
}

PACS numbers: 64.60.Ht, 64.60.Cn, 05.70.Ln, 05.70Fh

Shortened version of the title: The short-time behavior of kinetic spherical model

\begin{abstract}
The kinetic spherical model with long-ranged interactions and an arbitrary initial order $m_{0}$ quenched from a very high temperature to $T\left(\leq T_{c}\right)$ is solved. In the short-time regime, the bulk order increases with a power law in both the critical and phase-ordering dynamics. To the latter dynamics, a power law for the relative order $m_{r} \sim-t^{-k}$ is found in the intermediate time-regime. The short-time scaling relations of small $m_{0}$ are generalized to an arbitrary $m_{0}$ and all the time larger than $t_{m i c}$. The characteristic functions $\varphi\left(b, m_{0}\right)$ for the scaling of $m_{0}$ and $\epsilon\left(b, T^{\prime}\right)$ for $T^{\prime}=T / T_{c}$ are obtained. The crossover between scaling regimes is discussed in detail.
\end{abstract}

Keywords: spherical model; short-time dynamics; phase ordering

* Supported by the National Natural Science Foundation of China under the project 19772074 and by the Deutsche Forschungsgemeinschaft under the project Schu 95/9-1. 


\section{Introduction}

In recent years, the universal scaling in non-equilibrium states have attracted much attention. The phase-ordering process (POP) [1] and the short-time critical dyanmics (SCD) [2] are two fruitful examples.

In the critical dynamics, the short-time phenomena are those which happen at the times just after a microscopic time-scale $t_{m i c}$ needed for a system to forget its microscopic details, and much smaller than the macroscopic time scale $t_{m a c} \sim \tau^{-\nu z}$. Since the pioneer work of H.K. Janssen et al [3], universal short-time scaling has been found in a variety of different models (for a recent review, see [4]). It is believed that the singularity of the temporal correlation is essential to the short-time scaling. Therefore the scaling can emerge in the short-time regime of the evolution eventhough all spatial correlations are still short-ranged.

The dynamics to be considered here has no conservation law, which is sometimes called model A [5]. All critical exponents as well as the critical point can be fixed in the short-time regime [6, 6, 8]. This is important especially for Monte Carlo simulations because at the critical temperature a system needs infinite time to relax to equilibrium due to the critical slowing down. It is good to avoid doing measurements in equilibrium states. Therefore, it is interesting to investigate the connection between the short-time regime and the long-time regime and the crossover of scaling patterns from one regime to another.

The power law increase of order in the SCD is valid only in the case of a small initial order-parameter $m_{0}$. The general case of an arbitrary $m_{0}$ has been discussed in [9]. The initial state with non-zero $m_{0}$ is off-critical. A characteristic function must be introduced to describe the moving of $m_{0}$ in the scale transformation. With the characteristic function, the scaling law can be extended to a large $m_{0}$ and all $t \gg t_{m i c}$. As far as we know, only the numerical evidence has been found for this characteristic function. In this paper, the soluble kinetic spherical model (KSM) will provide a concrete example.

Instead of a quench to the critical temperature $T_{c}$ in the SCD, the system in the POP is quenched to a temperature lower than the critical one. For a system quenched from a symmetric initial state, the final equilibrium is never achieved since the ergodicity is broken in the thermodynamics. Instead, the length-scale of ordered regions grows with time as the different broken- 
symmetry phases compete to select the equilibrium state. This temporal singularity should also be the essence of the ordering scaling. It has been found both experimentally and theoretically [10] that the POP at late times can be described by a single characteristic length-scale $L(t) \sim t^{\rho}$, reflecting the self-similarity of domain patterns at different times.

If the initial state breaks the symmetry, as the case of $m_{0} \neq 0$ to be discussed here, the equilibrium can be achieved. A natural question is how long the phase-ordering scaling will last. The non-zero $m_{0}$ generates a timescale $t_{i}$ which characterizes the most interesting short-time regime where we will see new scaling patterns. Since $t_{i} \rightarrow \infty$ as $m_{0} \rightarrow 0$, the so-called short-time regime in fact can persist to a very long time.

The SCD and the POP are governed by two different fixed points, i.e., $T_{c}$ and $T=0$ respectively, therefore they have different scaling laws. It will be interesting to see how the POP crosses over to the SCD as $T$ grows from 0 to $T_{c}$. In the context of POP, besides $t_{i}$ there is another time-scale $t_{\mu}$ given by the non-zero temperature. In this paper, we will introduce a characteristic function to describe the temperature dependence of the process. Since the critical exponents can not change smoothly, there must be a crossover region that separates the SCD scaling domain from the scaling domain of POP. We will estimate the domain boundary through a self-consistency of the solution.

To obtain explicit analytical results, we confine ourself to the soluble KSM with or without long-ranged interactions. The KSM is a generalization to the static spherical model [11], to which a model A dynamics is given by the Langevin equation. The static spherical model has been proven to be equivalent to the large- $n$ limit $n$-vector model (LLNM) in static properties [12]. When the dynamics is included, they should be still equivalent in the thermodynamic limit since the fluctuation of $\left\langle\phi^{2}\right\rangle$ in the LLNM can be neglected. The LLNM being a soluble kinetic model with a dimensionality higher than one has been extensively studied by many authors. The SCD of the LLNM with the short-ranged interaction and small $m_{0}$ is well-known [3, 13, 14. To the symmetric POP, i.e., $m_{0}=0$, many results have been obtained for the model with either the short-ranged or the long-ranged interaction [15, 16, 17, 18]. We will use the language of KSM to present our new results originated from the non-symmetry initial state, i.e., an arbitrary $m_{0}$. The KSM has the domain-wall picture which is helpful for understanding the ordering process and the crossover from the POP to the SCD.

In this paper, we will concentrate on the consequences of non-symmetric 
initial states. The time-dependent order parameter $m(t)$ as well as the response propagator and correlation function are calculated. The dynamic behavior of the symmetric KSM with short-ranged interaction in the long-time regime was already studied in [19], where no trace of the initial condition is left. In the case of non-zero $m_{0}$, the order-parameter has non-equilibrium values in the short-time regime after the system is quenched from a very high temperature. It will show rich scaling patterns governed by the double time-scales $t_{i}$ and $t_{\mu}$.

It has been known that the critical exponents can be modified by the interaction range. How the characteristic critical exponent $\theta$ or $\theta^{\prime}$ depends on the interaction range has not been explicitly shown. But from the general consideration of that the longer interaction range and higher dimension are both in favor of the spacial correlation, the long interaction range will decrease the exponent $\theta^{\prime}$. We will show that is just the case in the KSM. Particularly we show that the interaction-range parameter $\sigma$ and the dimensionality $d$ enter the time-dependent order-parameter $m(t)$ always in the combination $d / \sigma$.

The remainder of this paper is organized as follows. In section 2 the kinetic spherical model is introduced. Laplace transformation to $m(t)$ is established in section 3. Solutions and scaling behaviors at $T=T_{c}$ and $T<T_{c}$ are obtained in section 4 and section 5 , respectively. In section 6 , a concrete example of $d / \sigma=3 / 2$ is given. Our conclusions and discussions will be given in the last section.

\section{The Model}

The Hamiltonian of the spherical model is

$$
H=\frac{\alpha}{2} \sum_{i} S_{i}^{2}-\frac{\beta}{2} \sum_{i j} J_{i j} S_{i} S_{j}
$$

with the constraint

$$
\sum_{i} S_{i}^{2}=N
$$

where $i, j$ are labels of lattice sites, $N$ is the total number of spins; $\beta=\frac{1}{k_{B} T}$. In the dynamic process, $\alpha$ is a time-dependent Lagrange multiplier corresponding to the constraint. Joyce [20] first studied the static spherical model 
with long-ranged ferromagnetic interactions. In a $d$-dimensional lattice,

$$
J_{i j}=J_{0} r_{i j}^{-(d+s)} / \sum_{j} r_{i j}^{-(d+s)}
$$

with $0<s<2$ for long-ranged interactions, while $s>2$ for short-ranged interactions. Where $r_{i j}$ is the distance between the sites $i$ and $j$.

The Langevin equation for this constrained spin system is

$$
\frac{\partial S_{i}}{\partial t}=-\lambda \alpha S_{i}+\lambda \beta \sum_{j} J_{i j} S_{j}+\eta_{i}
$$

where $\lambda$ is the kinetic coefficient and $\eta_{i}$ being a Gaussian white noise characterized by

$$
<\eta_{i}(t)>=0, \quad<\eta_{i}(t) \eta_{j}\left(t^{\prime}\right)>=2 \lambda \delta_{i j} \delta\left(t-t^{\prime}\right)
$$

where angle brackets mean average over the noise.

Since the constraint is applied to the whole dynamic process, at any time $t$, there is a consistency condition

$$
<\sum_{i} S_{i} \frac{\partial S_{i}}{\partial t}>=0
$$

The time-dependent order-parameter $m(t)=\frac{1}{N}<\sum_{i} S_{i}>$ is in the translationally invariant case equal to $\left\langle S_{i}>\right.$. Taking the average over the noise in (3), one has

$$
\frac{\partial m(t)}{\partial t}=-\lambda \tau(t) m(t)
$$

where $\tau(t)=\alpha(t)-\beta J_{0}$.

The fluctuations of spins are defined to be $\widetilde{S}_{i}=S_{i}-m(t)$. In the momentum space, one has

$$
\widetilde{S}(p, t)=\frac{1}{\sqrt{N}} \sum_{i} \widetilde{S}_{i} e^{i \mathbf{p} \cdot \mathbf{r}_{\mathbf{i}}}, \quad \eta(p, t)=\frac{1}{\sqrt{N}} \sum_{i} \eta_{i} e^{i \mathbf{p} \cdot \mathbf{r}_{\mathbf{i}}}
$$

with $r_{i}$ the position vector of site $i$, and

$$
J(p)=\sum_{j} J_{i j} e^{i \mathbf{p} \cdot \mathbf{r}_{\mathbf{i j}}}
$$


with $J(0)=J_{0}$. Corresponding to (3), the dynamic equation for $\widetilde{S}(p, t)$ is

$$
\frac{\partial \widetilde{S}(p, t)}{\partial t}=-\lambda(\tau(t)+\Delta(p)) \widetilde{S}(p, t)+\eta(p, t)
$$

where $\Delta(p)=\beta\left(J_{0}-J(p)\right)$. The consistency condition gives

$$
\begin{aligned}
\tau(t) & =\tau_{\text {sub }}+\beta J_{0}\left[m^{2}(t)-1\right]+\frac{\beta}{N} \sum_{p} J(p)<\widetilde{S}(-p, t) \widetilde{S}(p, t)> \\
& +\frac{1}{\lambda N} \sum_{p}<\widetilde{S}(-p, t) \eta(p, t)>
\end{aligned}
$$

where the first term comes from the mass subtraction which guarantees $\tau_{c}(\infty)=0$ at the critical point. It can be shown that $\tau_{\text {sub }}=1$. Due to causality, the last term of (8) is zero.

By solving (7), it is not difficult to obtain the response propagator

$$
G_{p}\left(t, t^{\prime}\right)=\frac{1}{2 \lambda}<\widetilde{S}(-p, t) \eta\left(p, t^{\prime}\right)>=\Theta\left(t-t^{\prime}\right) e^{-\lambda \Delta(p)\left(t-t^{\prime}\right)-\lambda \int_{t^{\prime}}^{t} d t^{\prime \prime} \tau\left(t^{\prime \prime}\right)}
$$

with the Heaviside step function $\Theta\left(t-t^{\prime}\right)=1$ for $t>t^{\prime}$, otherwise $\Theta\left(t-t^{\prime}\right)=$ 0 ; and the full correlation function (correlation function including the initial correlation)

$$
\begin{aligned}
\widetilde{C}_{p}\left(t, t^{\prime}\right) & =<\widetilde{S}(p, t) \widetilde{S}\left(-p, t^{\prime}\right)> \\
& =<\widetilde{S}(p, 0) \widetilde{S}(-p, 0)>G_{p}(t, 0) G_{-p}\left(t^{\prime}, 0\right)+C_{p}\left(t, t^{\prime}\right)
\end{aligned}
$$

with the correlation function

$$
C_{p}\left(t, t^{\prime}\right)=2 \lambda \int_{0}^{\infty} d t^{\prime \prime} G_{p}\left(t, t^{\prime \prime}\right) G_{p}\left(t^{\prime}, t^{\prime \prime}\right)
$$

where $<\widetilde{S}(p, 0) \widetilde{S}(-p, 0)>$ is given by the initial state. Consider a system initially at a temperature $T \gg T_{c}$ with a given initial order-parameter $m_{0}$. Since initial correlations are short-ranged, one has $<\widetilde{S}(p, 0) \widetilde{S}(-p, 0)>=$ $\left(1-m_{0}^{2}\right)$. 


\section{Laplace transformation}

Introducing $f(t)=m^{-2}(t)$, from (6) and (8) one obtains a linear integrodifferential equation for $f(t)$

$$
\frac{\partial f(t)}{\partial t}=2 \lambda \beta J_{0}-2 \lambda\left(\beta J_{0}-1\right) f(t)+\frac{2 \lambda \beta}{N} \sum_{p} J(p) \widetilde{C}_{p}(t, t) f(t)
$$

For a $d$-dimensional cubic lattice,

$$
J(p)=J_{0} C_{d, s}(p) / C_{d, s}(0)
$$

with $C_{d, s}(p)=\sum_{l}|\mathbf{l}|^{-(d+s)} \cos (\mathbf{l} \cdot \mathbf{p})$ and $\mathbf{l} \cdot \mathbf{p}=l_{1} p_{1}+\ldots+l_{d} p_{d}$. The critical temperature is given by [20]

$$
\beta_{c} J_{0}=\frac{1}{N} \sum_{p}\left[1-C_{d, s}(p) / C_{d, s}(0)\right]^{-1}
$$

Define

$$
w(x)=\frac{1}{N} \sum_{p} \frac{1}{x+\Delta(p) /\left(\beta J_{0}\right)}
$$

then $\beta_{c} J_{0}=w(0)$. By Laplace transformation

$$
F(q)=\int_{0}^{\infty} d t f(t) e^{-q t}
$$

Equation (12) is transformed to

$$
F(q)=\frac{\frac{\beta J_{0}}{q}+\frac{1}{2 \lambda}\left(m_{0}^{-2}-1\right) w\left(\frac{q}{2 \lambda \beta J_{0}}\right)}{\beta J_{0}-w\left(\frac{q}{2 \lambda \beta J_{0}}\right)}
$$

Using properties of Laplace-transformation, it is easy to obtain

$$
\begin{gathered}
\lim _{q \rightarrow \infty} q F(q)=m_{0}^{-2} \\
\lim _{q \rightarrow 0} q F(q)=m^{-2}(\infty)=\left(1-T / T_{c}\right)^{-1}
\end{gathered}
$$

Hence the initial condition is satisfied, and the infinite time limit correctly recovers the equilibrium result. From (17), one can also extract the critical exponent $\beta=1 / 2$. 
For small $p$, one has

$$
1-C_{d, s}(p) / C_{d, s}(0)=C p^{\sigma}
$$

where the constant $C$ depends only on $d$ and $s$. The parameter $\sigma$ denotes an effective potential-range defined by [21], with $\sigma=s$ for $0<s<2$ and $\sigma=2$ for $s>2$.

From here after, we suppose $d / 2<\sigma<d$. For the infinite system, the sum in (14) is replaced by an integral. Combining (13) with (18), in the continuum-limit one has

$$
w(x)=w(0)-D x^{d / \sigma-1}
$$

where the constant $D$ again depends only on $\sigma$ and $d$ and is positive. Then (15) becomes

$$
F(q)=\frac{\frac{\beta J_{0}}{q}+\frac{\beta J_{0}}{2 \lambda}\left(m_{0}^{-2}-1\right)}{\left.\left(\beta-\beta_{c}\right)\right) J_{0}+D\left(\frac{q}{2 \lambda \beta J_{0}}\right)^{d / \sigma-1}}-\frac{1}{2 \lambda}\left(m_{0}^{-2}-1\right)
$$

Since the microscopic details have been skipped in the continuum limit, (20) does not give the correct initial value. Instead, it gives $f(0)=\infty$ corresponding to $m(0)=0$. Therefore (20) is only valid for $t$ larger than a microscopic time-scale $t_{m i c}$. The last term of (20) only has contribution at $t=0$, therefore it can be dropped for the regime $t>t_{m i c}$.

Notably, if one rescales the kinetic coefficient $\lambda$ and the initial order $m_{0}$ as following

$$
\begin{gathered}
\lambda^{\prime}=\lambda D_{c}^{\frac{1}{1-d / \sigma}} \\
m_{0}^{\prime}=\left[D_{c}^{\frac{1}{1-d / \sigma}}\left(m_{0}^{-2}-1\right)+1\right]^{-1 / 2}
\end{gathered}
$$

with $D_{c}=D /\left(\beta_{c} J_{0}\right)^{d / \sigma}$, then $F(q)$ depends on the dimension $d$ and the interaction-range parameter $\sigma$ through their ratio $k=d / \sigma$, i.e., for $t>t_{\text {mic }}$,

$$
F(q)=\frac{\frac{1}{q}+\frac{1}{2 \lambda^{\prime}}\left(m_{0}^{\prime-2}-1\right)}{\mu+(1-\mu)^{k}\left(\frac{q}{2 \lambda^{\prime}}\right)^{k-1}}
$$

where $\mu=\left(T_{c}-T\right) / T_{c}$ is the reduced temperature. $D_{c}$ is a non-universal constant depending on $d, \sigma$ and the regularization scheme. As one will see in the next section, (22) follows from a rescaling of time by a factor $D_{c}^{\frac{1}{1-k}}$.

Equation (23) is the starting point of the following discussions. 


\section{4. $T=T_{c}$}

At the critical point, $\mu=0$, the Laplace back-transformation of (23) is easily carried out. The solution for the order parameter at the critical point is

$$
m(t)= \pm\left[\frac{\Gamma(k)}{\left(2 \lambda^{\prime}\right)^{k-1} t_{i}}\right]^{1 / 2}\left(1+t / t_{i}\right)^{-1 / 2} t^{1-\frac{k}{2}}
$$

where $t_{i}$ is a characteristic time-scale of the short-time regime, which depends on the initial condition,

$$
t_{i}=\frac{(k-1)\left(m_{0}^{-2}-1\right)}{2 \lambda}=\frac{(k-1)\left(m_{0}^{\prime-2}-1\right)}{2 \lambda^{\prime}}
$$

It is invariant in the combined transformation of (21) and (22). The sign of $m(t)$ can be found from the symmetry of the initial state. The $|m(t)|$ firstly increases then it relaxes to zero. The maximum is at $t=\frac{2-k}{k-1} t_{i}$, it is propotional to $t_{i}$.

For small enough $m_{0}$, the $t_{i}$ could be much larger than $t_{m i c}$. Then in the regime $t_{m i c}<t \ll t_{i}$ one can observe the power-law order increasing suggested by Janssen et al [3],

$$
m(t) \sim t^{\theta^{\prime}}
$$

with the exponent $\theta^{\prime}=1-\frac{k}{2}$. This regime is called the critical initial slip. A standard explanation to the increase of the order is that the orderparameter follows a mean-field ordering process because $T_{c}<T_{c}^{m . f .}$ as long as correlations are short-ranged in the critical initial slip. However the exponent $\theta^{\prime}$ is non-trivial. As we will see in the next section, it is different from the corresponding exponent for the POP.

In the long-time regime, $t \gg t_{i}$,

$$
m(t)=\left[\frac{\Gamma(k)}{\left(2 \lambda^{\prime}\right)^{k-1}}\right]^{1 / 2} t^{-\frac{k-1}{2}}
$$

The dependence on the initial condition disappeares as expected. Comparing with the well-known nonlinear relaxation scaling law, one obtains $\frac{\beta}{\nu z}=\frac{k-1}{2}$.

When $m_{0} \neq 0$, the initial state is not at the fixed point. However, as proposed by Zheng [9], the short-time critical scaling hypothesis could be 
generalized to an arbitrary $m_{0}$ by introducing a characteristic function of $m_{0}$ and the scaling factor $b$.

As the length is rescaled by a factor $b$, supposing $t_{i}$ is rescaled by $b^{-z}$ just like a time, then $m(t)$ given by (24) satisfies the following scaling relation

$$
m\left(t, m_{0}\right)=b^{-\frac{\beta}{\nu}} m\left(b^{-z} t, \varphi\left(b, m_{0}\right)\right)
$$

where $\varphi\left(b, m_{0}\right)$ is the characteristic function, which describes how the initial order changes in the scale transformation. Its explicit form can be deduced from the scaling of $t_{i}$

$$
\varphi\left(b, m_{0}\right)=\left[b^{-z}\left(m_{0}^{-2}-1\right)+1\right]^{-\frac{1}{2}}
$$

The effective dimension of the initial order $m_{0}$ is defined by

$$
x_{0}\left(b, m_{0}\right)=\frac{\ln \left[\varphi\left(b, m_{0}\right) / m_{0}\right]}{\ln b}
$$

As expected, as $m_{0} \rightarrow 0, \varphi\left(b, m_{0}\right)=b^{z / 2} m_{0}$, so that the initial order has an anomalous dimension $x_{0}=\frac{z}{2}$ and the power law (26) is recovered. When $m_{0}$ takes its maximum $m_{0}=1, \varphi=1$ and $x_{0}=0$. In this case, $t_{i}=0$, hence only the nonlinear relaxation scaling law, $m(t) \sim t^{-\frac{\beta}{\nu z}}$, is observed. Figure 1 plots $x_{0}$ versus $m_{0}$ for $b=2$ and $b=4$. It is qualitatively consistent with the numerical results of Ising model [⿴] , for which $x_{0}\left(b, m_{0}\right)$ is a monotonously decreasing function of $m_{0}$. This appeares to be reasonable by recalling that the spherical model is just a very rough approximation of the Ising model.

Substituting (24) into (6), one gets $\tau(t)=\frac{k-2}{2 \lambda t}+\frac{1}{2 \lambda\left(t+t_{i}\right)}$. This result together with (9) gives the response propagator

$$
G_{p}\left(t, t^{\prime}\right)=\Theta\left(t-t^{\prime}\right)\left(\frac{t}{t^{\prime}}\right)^{(2-k) / 2}\left(\frac{t^{\prime}+t_{i}}{t+t_{i}}\right)^{1 / 2} e^{-\widetilde{\lambda} p^{\sigma}\left(t-t^{\prime}\right)}
$$

where $\tilde{\lambda}=\lambda C \beta J_{0}$ with $\beta=\beta_{c}$ in this case. The correlation function (11) is given by

$$
\begin{aligned}
C_{p}\left(t, t^{\prime}\right)= & 2 \lambda t^{\prime}\left(\frac{t}{t^{\prime}}\right)^{\frac{2-k}{2}}\left(\frac{t^{\prime}+t_{i}}{t+t_{i}}\right)^{\frac{1}{2}} e^{-\widetilde{\lambda} p^{\sigma}\left(t-t^{\prime}\right)} \\
& \cdot \int_{0}^{1} d y(1-y)^{k-2} \frac{t^{\prime}-t^{\prime} y+t_{i}}{t^{\prime}+t_{i}} e^{-2 \widetilde{\lambda} p^{\sigma} t^{\prime} y}
\end{aligned}
$$


When $t \ll t_{i}$ and $t^{\prime} \ll t_{i}$, (31) has a scaling form,

$$
G_{p}\left(t, t^{\prime}\right)=p^{-2+\eta+z}\left(\frac{t}{t^{\prime}}\right)^{\theta} h\left(p^{z}\left(t-t^{\prime}\right)\right)
$$

with $h(x)$ being a scaling function, as suggested in [2]. The exponents $\theta=$ $1-\frac{k}{2}, z=\sigma$ and $\eta=2-\sigma$ can be read out. The values of $z$ and $\eta$ agree with the corresponding results in [22, 23, 24] and [20, 25, 26]. Two exponents $\theta$ and $\theta^{\prime}$ are equal to each other. For short-ranged interactions $\sigma=2$, they coincide with the results of LLNM [3, 27, 13, 14].

When $t=t^{\prime} \ll t_{i}$, we get the equal-time correlation function

$$
C_{p}(t, t)=\frac{2 \lambda t e^{-2 \widetilde{\lambda} p^{\sigma} t}}{k-1}\left(1+O\left(2 \widetilde{\lambda} p^{\sigma} t\right)\right)
$$

It has the scaling form [3, 13, 14, 15, 28] $C_{p}(t, t)=p^{-2+\eta} g\left(p \xi_{c}(t)\right)$ with $g(x)$ being another scaling function. Where $\xi_{c}(t) \sim t^{1 / \sigma}$ is a characteristic lengthscale. Physically, it should be related to the correlation length.

When $t, t^{\prime} \gg t_{i}$ and $2 \widetilde{\lambda} p^{\sigma} t^{\prime}$ large, the propagator and equal-time correlation function reads as

$$
\begin{gathered}
G_{p}\left(t, t^{\prime}\right)=\Theta\left(t-t^{\prime}\right)\left(\frac{t}{t^{\prime}}\right)^{-\frac{k-1}{2}} e^{-\widetilde{\lambda} p^{\sigma}\left(t-t^{\prime}\right)} \\
C_{p}(t, t)=\frac{p^{-\sigma}}{C \beta_{c} J_{0}}\left[1-\frac{k-1}{2 \widetilde{\lambda} p^{\sigma} t}+O\left(\frac{1}{\left(\widetilde{\lambda} p^{\sigma} t\right)^{2}}\right)\right]
\end{gathered}
$$

By use of the characteristic function (29), the response propagator and correlation function have general scaling forms which are valid for the times up to $t_{m i c}$ and for an arbitrary $m_{0}$,

$$
\begin{aligned}
G_{p}\left(t, t^{\prime}, m_{0}\right) & =p^{-2+\eta+z} \widetilde{h}\left(p \xi_{c}(t), p \xi_{c}\left(t^{\prime}\right), \varphi\left(p^{-1}, m_{0}\right)\right) \\
C_{p}\left(t, t^{\prime}, m_{0}\right) & =p^{-2+\eta} \widetilde{g}\left(p \xi_{c}(t), p \xi_{c}\left(t^{\prime}\right), \varphi\left(p^{-1}, m_{0}\right)\right)
\end{aligned}
$$

where $\widetilde{h}$ and $\widetilde{g}$ are scaling functions. The significance of the characteristic function is to connect the short-time regime and the long-time regime so that for each thermodynamic quantity only one scaling function is needed for the whole physical interesting time regime. 


\section{5. $T<T_{c}$}

In the case of $T<T_{c}$, the system undergoes an ordering process which is governed by the fixed point $T=0$. For large $\beta$, i.e., $\mu \rightarrow 1$, one can expand the denominator of (23) to get

$$
F(q)=\mu^{-1}\left[\frac{1}{q}-\frac{2 \lambda^{\prime}}{\mu}\left(\frac{1-\mu}{2 \lambda^{\prime}}\right)^{k} q^{k-2}-\frac{m_{0}^{\prime-2}-1}{\mu}\left(\frac{1-\mu}{2 \lambda^{\prime}}\right)^{k} q^{k-1}\right]
$$

The asymptotic solution for the bulk order is obtained by the Laplace backtransformation

$$
m(t)=m(\infty)\left[1-\left(1-\frac{t_{i}}{t}\right)\left(\frac{t}{t_{\mu}}\right)^{1-k}\right]^{-1 / 2}
$$

where $t_{\mu}=\left[\frac{2 \lambda^{\prime}}{|\mu| \Gamma(2-k)}\left(\frac{1-\mu}{2 \lambda^{\prime}}\right)^{k}\right]^{\frac{1}{k-1}}$. Again for a fixed $k$, the dependence on $d$ and $\sigma$ can be absorbed by a proper rescaling of the time.

The $t_{\mu}$ is a temperature-dependent time-scale. The corresponding lengthscale should be the domain-width $\xi_{d w} \sim t_{\mu}^{1 / z}$. The POP scaling only could survive when $\xi_{d w}$ is smaller than the domain-size which grows with time as $\xi \sim t^{1 / z}$. That implies $t_{\mu}$ should be smaller than a characteristic time of the phase-ordering regime. From the consistency of (39) we do find a constraint for $t_{\mu}$

$$
\frac{t_{\mu}}{t_{i}}<\frac{k^{\frac{k}{k-1}}}{k-1} .
$$

Replacing $t_{\mu}$ by its explicit expression, one has

$$
\left(1-\frac{T}{T_{c}}\right)\left(\frac{T_{c}}{T}\right)^{k}>\frac{1}{k \Gamma(2-k)}\left(\frac{k-1}{2 \lambda^{\prime} k t_{i}}\right)^{k-1}
$$

The r.h.s is a positive constant. The l.h.s is a monotonously decreasing function with respect to $T$, which has zero value at $T=T_{c}$. Hence there is a boundary temperature $T_{b}<T_{c}$. The ordering scaling appears in the regime $0<T<T_{b}$. The smaller $m_{0}$, the larger $T_{b}$. It tends to $T_{c}$ as $m_{0} \rightarrow 0$.

As $t \ll t_{i}$, one has

$$
m(t)=m(\infty)\left[1+\left(\frac{t_{i}^{\prime}}{t}\right)^{k}\right]^{-\frac{1}{2}}
$$


where $t_{i}^{\prime}=t_{\mu}\left(t_{i} / t_{\mu}\right)^{1 / k}$ is a time-scale that characterizes the short-time ordering behavior. In the symmetry case, $t_{i}^{\prime}=\infty$ so $m(t)=0$. No information about the POP can be extracted from the bulk order-parameter. With a non-symmetric initial state however, the process has various scaling behaviors manifested by $m(t)$ due to the competition of two time-scales. From the self-consistency condition mentioned above, one can show that in the scaling regime, $t_{i}$ is at least larger than $t_{i}^{\prime} / 2$. There will be three different scaling regimes.

(a) $t_{m i c}<t \ll t_{i}^{\prime}$ and $t_{i}$

This is the extensively studied regime in the symmetric POP. In literatures, sometimes it is called the late time stage. There is no contradiction because in the symmetry case $t_{i}$ and $t_{i}^{\prime}$ are infinite. The late-time simply means $t>t_{\text {mic }}$.

For small $m_{0}, t_{i}^{\prime}$ and $t_{i}$ can be much larger than $t_{m i c}$. Then in this regime, one can observe a power law increase of the order-parameter

$$
m(t)=m(\infty)\left(\frac{t}{t_{i}^{\prime}}\right)^{\theta_{T}^{\prime}}
$$

with $\theta_{T}^{\prime}=k / 2$. This is the initial increase in the POP.

The response propagator when $t \ll t^{\prime}{ }_{i}$ and $t_{i}$ is

$$
G_{p}\left(t, t^{\prime}\right)=\Theta\left(t-t^{\prime}\right)\left(\frac{t}{t^{\prime}}\right)^{\theta_{T}} e^{-\widetilde{\lambda}^{\sigma}\left(t-t^{\prime}\right)}
$$

with $\theta_{T}=k / 2$. We note that [28] got the exponent $\theta_{T}$ at $\sigma=2$ for the response propagator in the study of the domain growth.

(b) $t_{i}^{\prime} \ll t \ll t_{i}$

The existence of this regime depends on both the temperature $T$ and the initial order $m_{0}$. The self-consistency of (39) implies $\frac{t_{i}^{\prime}}{t_{i}}<k(k-1)^{(1-k) / k}$. As $T \rightarrow 0, t^{\prime}{ }_{i}$ tends to zero while $t_{i}$ does not change. On the other hand, as far as $k>1, t_{i}$ tends to infinite faster than $t^{\prime}{ }_{i}$ as $m_{0} \rightarrow 0$. Therefore, this regime exists if $m_{0}$ is small but not exactly zero and the temperature is low.

Define a relative order with respect to the equilibrium order,

$$
m_{r}\left(t, T^{\prime}, m_{0}\right)=\frac{m(t)-m(\infty)}{m(\infty)}
$$

where $T^{\prime}=T / T_{c}$. One can easily obtain from (42)

$$
m_{r}(t)=-\frac{1}{2}\left(\frac{t_{i}^{\prime}}{t}\right)^{k}
$$


The $m_{r}$ asymptotically follows a power law.

When $t_{i}^{\prime} \ll t \ll t_{i}$ while $t^{\prime} \ll t_{i}^{\prime}$, the response propagator is

$$
G_{p}\left(t, t^{\prime}\right)=\Theta\left(t-t^{\prime}\right)\left(\frac{t_{i}^{\prime}}{t^{\prime}}\right)^{\theta_{T}} e^{-\widetilde{\lambda} p^{\sigma}\left(t-t^{\prime}\right)}
$$

The time-scale $t_{i}^{\prime}$ enters the propagator.

Another case is $t_{i}{ }_{i} \ll t^{\prime}, t \ll t_{i}$. The response propagator has an asymptotic form

$$
G_{p}\left(t, t^{\prime}\right)=\Theta\left(t-t^{\prime}\right) e^{-\widetilde{\lambda} p^{\sigma}\left(t-t^{\prime}\right)}
$$

The corresponding equal-time correlation function is

$$
C_{p}(t, t)=\frac{\lambda}{\widetilde{\lambda} p^{\sigma}}\left(1-e^{-2 \widetilde{\lambda} p^{\sigma} t}\right)
$$

It exponentially tends to the structure function of the equilibrium state.

(c) $t \gg t_{i}$

Notably, in the long-time regime (including all time if $\left.m_{0}=1\right),|m(t)|$ approaches $|m(\infty)|$ from above if $m_{0} \neq 0$, and

$$
m_{r}(t)=\frac{1}{2}\left(\frac{t_{\mu}}{t}\right)^{k-1}
$$

The asymptotic power law has an exponent $\theta_{1}^{\prime}=1-k$, which is different from $-\beta / \nu z=\frac{1-k}{2}$ in the critical nonlinear relaxation. This is in agreement with the results of [19, 29] in the special case of $\sigma=2$. It can be understood since two processes are governed by different fixed points.

In the general case, $0<T<T_{b}$ and $m_{0} \neq 0$, the dimension of the order-parameter is scale-dependent. As one has seen in the cases (b) and (c), a more suitable scaling operator is the relative order $m_{r}$. There exist two off-critical parameters, $T^{\prime}$ and $m_{0}$. They will be running versus the scale in a scale transformation. Therefore one needs two characteristic functions. The characteristic function for $m_{0}$ has been given in (29). The charateristic function for $T^{\prime}$ can be obtained by requiring $t_{\mu}$ scaling as $t$, then one can have a general scaling relation,

$$
m_{r}\left(t, T^{\prime}, m_{0}\right)=m_{r}\left(b^{-z} t, \epsilon\left(b, T^{\prime}\right), \varphi\left(b, m_{0}\right)\right)
$$

The characteristic function $\epsilon\left(b, T^{\prime}\right)$ satisfies the following equation

$$
\frac{\epsilon^{k}\left(b, T^{\prime}\right)}{1-\epsilon\left(b, T^{\prime}\right)}=b^{-z(k-1)} \frac{T^{\prime k}}{1-T^{\prime}}
$$


It can be easily checked that $\epsilon(b, 0)=0, \epsilon(b, 1)=1, \epsilon\left(1, T^{\prime}\right)=1$, and $\epsilon\left(\infty, T^{\prime}\right)=0$. In fact (49) is valid for all temperature (if $m(\infty)$ is replaced by $\sqrt{|\mu|}$ when $T>T_{c}$ ) as one will see from the example of the next section. One can again define the effective dimension of $T^{\prime}$ as

$$
y\left(b, T^{\prime}\right)=\frac{\ln \left(\epsilon\left(b, T^{\prime}\right) / T^{\prime}\right)}{\ln \left(T^{\prime}\right)}
$$

When $T \rightarrow 0$, one has a constant dimension for $T^{\prime}$, i.e., $y(b, 0)=-z \frac{k-1}{k}$. It is negative since $T=0$ is an attractive fixed point. For $k=3 / 2$ and $b=2,4, y\left(b, T^{\prime}\right)$ is plotted versus $T^{\prime}$ in Figure 2 . In the vicinity of the critical temperature, the scaling of the reduced temperature can be recovered from (50), i.e., $\mu(b)=1-\epsilon\left(b, T_{c}\right)=b^{z(k-1)} \mu$. The critical exponent $\nu=z(k-1)$ is obtained.

As in the critical dynamics, with the help of the characteristic functions, the response propagator and correlation function can be casted into scaling functions

$$
\begin{gathered}
G_{p}\left(t, t^{\prime}, T^{\prime}, m_{0}\right)=\widetilde{h}^{\prime}\left(p \xi(t), p \xi\left(t^{\prime}\right), \epsilon\left(p^{-1}, T^{\prime}\right), \varphi\left(p^{-1}, m_{0}\right)\right) \\
C_{p}\left(t, t^{\prime}, T^{\prime}, m_{0}\right)=p^{-\sigma} \widetilde{g}^{\prime}\left(p \xi(t), p \xi\left(t^{\prime}\right), \epsilon\left(p^{-1}, T^{\prime}\right), \varphi\left(p^{-1}, m_{0}\right)\right)
\end{gathered}
$$

Where the characteristic length $\xi(t) \sim t^{\rho}$ with $\rho=\frac{1}{\sigma}$ is the domain size [ [G]. Apart from some arbitrary coefficients, $\widetilde{h}^{\prime}$ and $\widetilde{g}^{\prime}$ are universal.

\section{Example of $k=3 / 2$}

In order to see the crossover more clearly, let us consider the concrete example of $k=3 / 2$. The 3 -dimensional model with the short-ranged interaction belongs to this case. A compact solution for $\mathrm{m}(\mathrm{t})$ is available.

(a) $T \leq T_{c}$

A direct Laplace back-transformation to (23) gives

$$
m(t)=m(\infty)\left[1+\frac{2 t_{i}}{\pi t_{\mu}} \sqrt{\frac{t_{\mu}}{t}}-\left(1+\frac{2 t_{i}}{\pi t_{\mu}}\right) e^{\frac{t}{\pi t_{\mu}}} \operatorname{erfc}\left(\sqrt{\frac{t}{\pi t_{\mu}}}\right)\right]^{-1 / 2}
$$

where $\operatorname{erfc}(x)=\frac{2}{\sqrt{\pi}} \int_{x}^{\infty} e^{-\tau^{2}} d \tau$ is the complementary error function. 
Taking the limit $\mu=0\left(T=T_{c}\right)$, the critical scaling of (24) for $k=3 / 2$ is recovered. In the other hand, for small $T, \mu \sim 1$, one has the ordering scaling for $k=3 / 2$ given in section 5 .

Since no expansions around $T=0$ have been made in obtaining $m(t)$, (53) is valid at temperatures equal and lower than $T_{c}$.

In Figure 3, we compare the results of (39) and (53). As the selfconsistency condition (40) implies, $m(t)$ of (39) graduately deviates from the correct results of (53) as the temperature increases, and becomes singular when it is bigger than certain temperature.

(b) $T>T_{c}$

For completeness, let us also write down the exponential relaxation in the high temperature phase. Since $\beta_{c}>\beta,(23)$ has a positive singular point $\left(\pi t_{\mu}\right)^{-1}$,

$$
F(q)=\frac{1}{|\mu|\left(\pi t_{\mu}\right)^{1 / 2}} \frac{1+2 t_{i} q}{q\left(q^{1 / 2}-\left(\pi t_{\mu}\right)^{-1 / 2}\right)}
$$

The Laplace back-transformation gives

$$
m(t)=\sqrt{|\mu|}\left[\frac{2 t_{i}}{\pi t_{\mu}} \sqrt{\frac{t_{\mu}}{t}}-1+\left(1+\frac{2 t_{i}}{\pi t_{\mu}}\right) e^{\frac{t}{\pi t_{\mu}}}\left(2-\operatorname{erfc}\left(\sqrt{\frac{t}{\pi t_{\mu}}}\right)\right)\right]^{-\frac{1}{2}}
$$

In the long-time regime, one has the familiar exponential decay

$$
m(t) \sim e^{-\frac{t}{2 \pi t \mu}}
$$

\section{Conclusion and discussions}

In summary, we solve the KSM with long-ranged interactions. The system is quenched from a very high temperature into either $T=T_{c}$ or $T<T_{c}$, with an arbitrary $m_{0}$. The bulk order as well as the response propagator and correlation function are calculated.

For small $m_{0}$, we recover the power law of the initial increase in the SCD. We obtain $\theta^{\prime}=\theta=1-\frac{k}{2}$. For the short-ranged interaction, they agree with the existing results.

The initial order increasing power law is also found in the POP, but with the exponent $\theta_{T}^{\prime}=k / 2$ different from that in the critical dynamics. As the symmetry is broken, a new scaling regime emerges. In the time range 
$t^{\prime}{ }_{i}<t<t_{i}$ the relative order has a power law $m_{r} \sim-t^{-k}$, while in the long-time regime $m_{r} \sim t^{-(k-1)}$.

We obtain the characteristic function of $m_{0}$ which enables us to generalize the critical short-time scaling relation to the whole time range and to an arbitrary $m_{0}$.

As in the SCD, the whole ordering process with broken symmetry can be described by universal scaling functions with the help of two characteristic functions. The one that arises from the off-critical temperature is given through the transcendent equation (50). The other one concerning the scaling property of $m_{0}$ is exactly the same as in the critical dynamics. This implies that the characteristic function $\varphi$ does not depend on the dynamics in the model under consideration. If the non-linear part of a dynamics is skipped, one will always obtain a monotonously decreasing effective dimension for the initial order. But it should not be a general conclusion. For the 3-state Potts model, instead a monotonously decreasing function the effective dimension $x_{0}\left(m_{0}\right)$ firstly increases then goes to zero [9]. Analytical calculations of characteristic functions from the Ising model or the Potts model remain as a challenge.

Although there is a similarity in the short-time behaviors between the SCD and POP, the physical pictures should be quite different for the two processes. In the critical dynamics, the characteristic length is the correlation length which is divergent as $t \rightarrow \infty$. Instead, it is the domain-size that tends to diverge in the POP. The domain-width will be controlled by the temperature. When the temperature approaches $T_{c}$ from below, the domainwidth grows as $t_{\mu}^{1 / z}$. As it gradually become comparable with the domainsize, the phase-ordering picture ceases to be valid. From the consistency condition of the solution for $m(t)$, we obtain the boundary temperature $T_{b}$ for the ordering scaling domain.

In the specific example of $k=3 / 2, m(t)$ is obtained for all temperatures. The ordering scaling can smoothly cross over to the critical scaling and then to the high-temperature phase. But at the crossover temperatures, there is no simple scaling law.

We find that the bulk time-dependent order has the special feature that it depends on $d$ and the interaction range-parameter $\sigma$ only through their ratio. The longer (shorter) the interaction-range is, the higher (lower) the effective dimension. It is physically plausible because the correlation can be strengthened (weakened) by either increasing (decreasing) the interaction- 
range or dimension.

The LLNM should have the same properties as those we find in the KSM. Therefore our results about the characteristic functions and the intermediatetime scaling in the POP can be used as the starting-point of finite- $n$ expansions.

We have investigated the KSM in the non-classical regime $d / 2<\sigma<$ $d$. In the classical regime $0<\sigma<d / 2$, the initial increase would be unobservable. When $\sigma \geq d$, the situation would be more complicated. At $\sigma=d$, there would be a logorithmic correction. We leave these for further investigation. Our results can also be extended to a finite-size lattice [13, 14].

Acknowledgements:Authors thank the very helpful discussions with B. Zheng and L. Schülke.

\section{References}

[1] A. J. Bray, Adv. Phys. 43, 357(1994)

[2] H.K. Janssen, in From Phase Transition to Chaos, edited by G. Györgyi, I. Kondor, L. Sasvári and T. Tél, Topics in Mondern Statistical Physics (World Scientific, Singapore, 1992)

[3] H. K. Janssen, B. Schaub, and B. Schmittmann, Z. Phys. B 73, $539(1989)$

[4] B. Zheng, Int. J. Mod. Phys. B 12, 1419(1998)

[5] P. I. Hohenberg and B. I. Halperin, Rev. Mod. Phys. 49,435(1977)

[6] G. G. Batrouni and A. Hansen, J. Phys. A:Math. Gen. bf 25, L1059 (1992)

[7] Z.B. Li, L. Schülke and B. Zheng, Phys. Rev. Lett. 74, 3396(1995)

[8] L. Schülke and B. Zheng, Phys. Lett. A233, 93(1997)

[9] B. Zheng, Phys. Rev. Lett. 77, 679(1996)

[10] A. J. Bray, preprint, cond-mat/9802183 
[11] T. H. Berlin and M. Kac, Phys. Rev. 86, 821(1952)

[12] H. E. Stanley, Phys. Rev. 176, 718(1968)

[13] H. W. Diehl and U.Ristschel, J. Stat. Phys. 73, 1(1993)

[14] H. W. Diehl and U.Ristschel, Phys. Rev. E 56, 5392(1995)

[15] A. Coniglio, P.Ruggiero, and M.Zannetti, Phys. Rev. E 50, 1046(1994)

[16] J. G. Kissner and A. J. Bray, J. Phys. A: Math. Gen. 26, 1571 (1993)

[17] K. Humayun and A. J. Bray, J. Phys. A: Math. Gen. 24, 1915 (1991)

[18] A. J. Bray, Phys. Rev. B 41, 6724(1990)

[19] C. Murakami and H. Tomita, Progr. Theor. Phys. 60, 699(1978)

[20] G. S. Joyce, Phys. Rev. 146, 349(1966)

[21] M. Suzuki, Prog. Theor. Phys. 49, 424 (1973); 49, 1017 (1973); 49, 1106 (1973); Phys. Lett. A42, 5 (1972)

[22] M. Suzuki, G. Igarashi, Prog. Theor. Phys. 49, 1070(1973)

[23] M. Suzuki, Phys. lett. A 43, 245(1973)

[24] M. Suzuki, Prog. Theor. Phys. 53, 97(1975)

[25] M. E. Fisher, S. Ma, and B. G. Nickel, Phys. Rev.Lett. 29, 917 (1972)

[26] E. Luijten and H. W. J. Blöte, Phys. Rev. B 56, 8945 (1997)

[27] K. Odering and H. K. Janssen, J. Phys. A: Math. Gen. 26, 3369(1993);

K. Odering and H. K. Janssen, J. Phys. A: Math. Gen. 26, 5295(1993)

[28] T. J. Newman and A. J. Bray, J. Phys. A: Math. Gen. 23, 4491(1990)

[29] R. Bausch and H. K. Janssen, Z. Phys B 37 ,163(1980) 


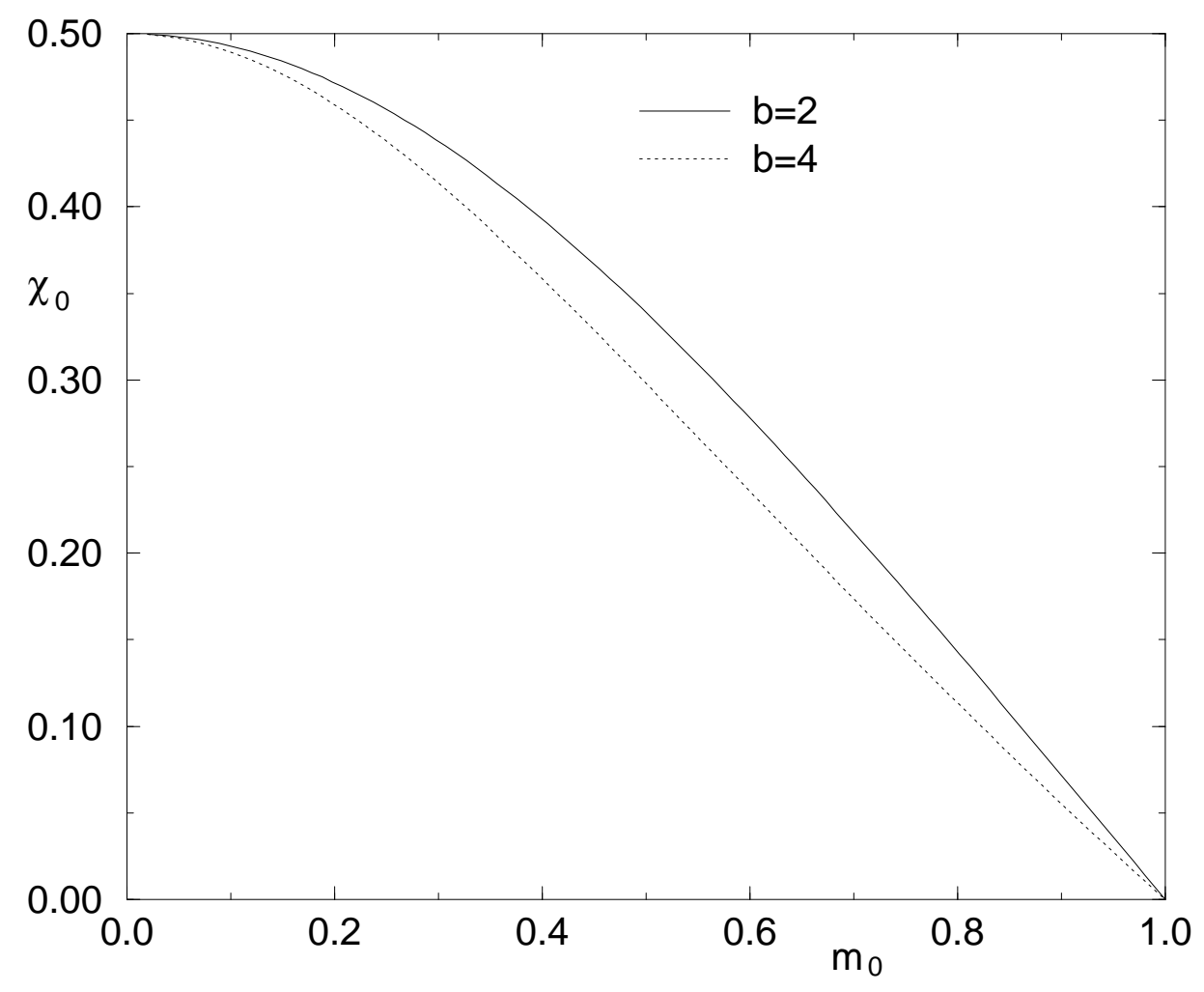

Figure 1: The effecitive dimension $x_{0}\left(b, m_{0}\right)$ versus $m_{0}$. The upper line is for $b=2$ while the lower line for $b=4$. 


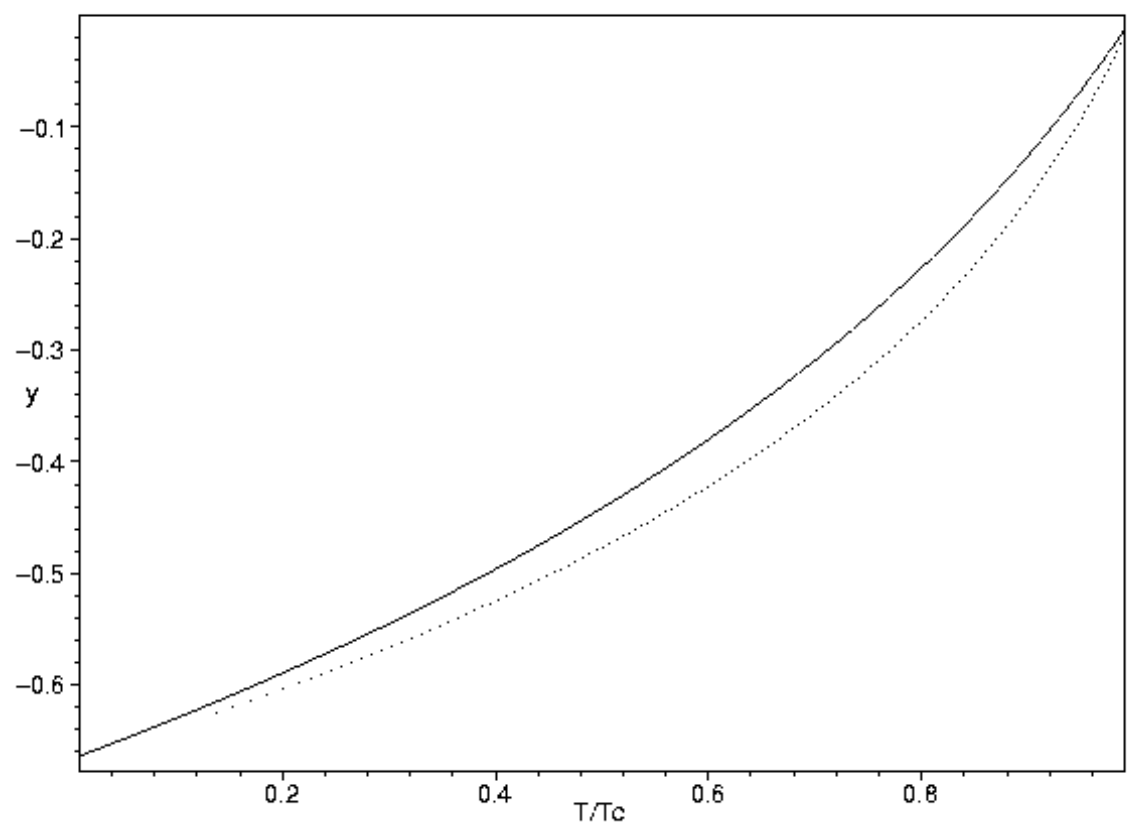

Figure 2: The effecitive dimension $y\left(b, T / T_{c}\right)$ versus $T / T_{c}$ with $k=3 / 2$. The upper line is for $b=2$ while the lower line for $b=4$. 


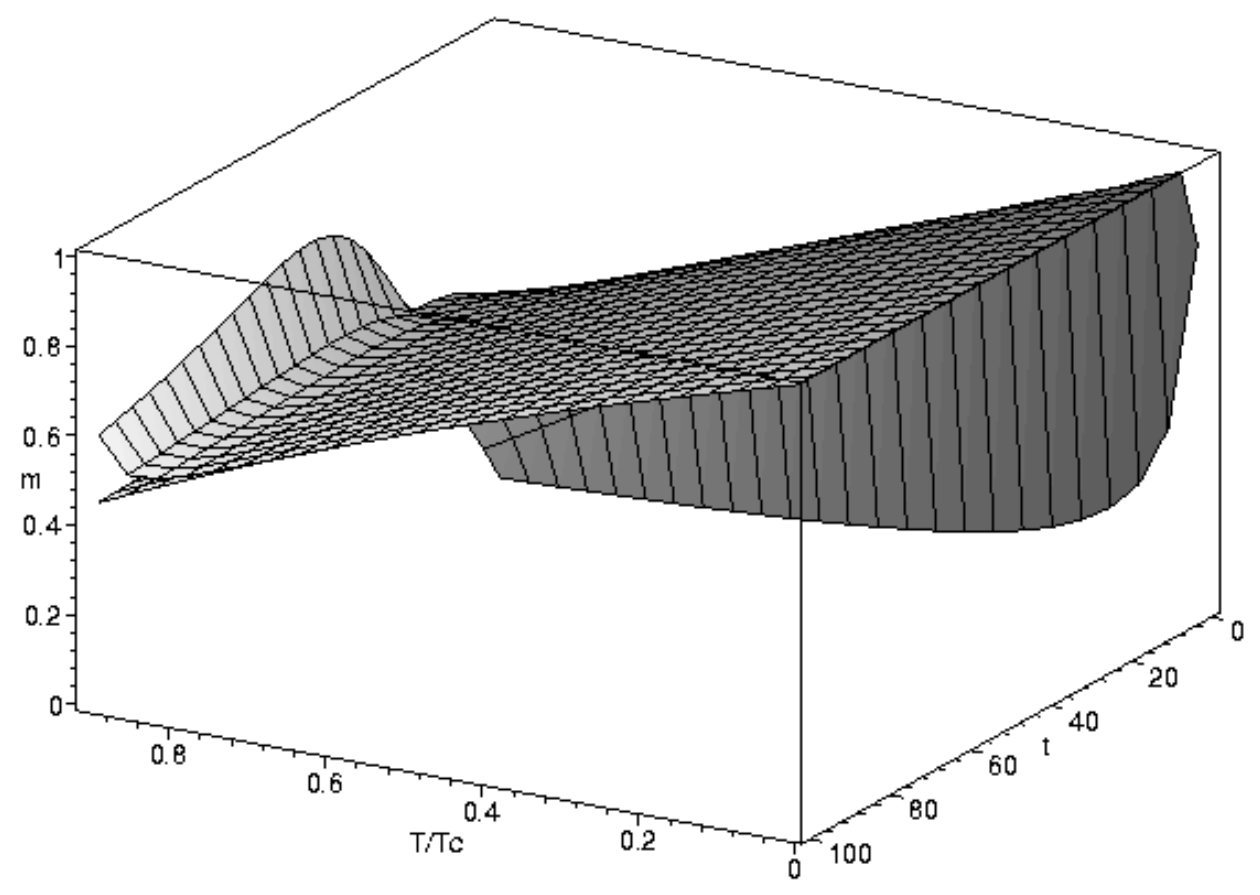

Figure 3: The time-dependent order $m(t)$ with $k=3 / 2$ of (39) and (53). In this figure, $t_{i}$ has been used as the time scale. The surface has infinite singularity in larger temperatures is the results of (39) obtained by the lowtemperature expansion, the other is the exact results of (53). 